\title{
Identidad profesional y formación del docente universitario: retos e incertidumbres
}

\section{Professional identity and training of the university teacher: challenges and uncertainties}

María josé Alcalá del Olmo ${ }^{1}$

Recibido: 14 de abril de 2019 Aceptado: 22 de mayo de 2019 Publicado: 31 de julio de 2019

To cite this article: Alcalá, M. J. (2019). Identidad profesional y formación del docente universitario: retos e incertidumbres.

Márgenes, Revista de Educación de la Universidad de Málaga, o (o), 18-37

DOI: http://dx.doi.org/10.24310/mgnmar.voio.6201

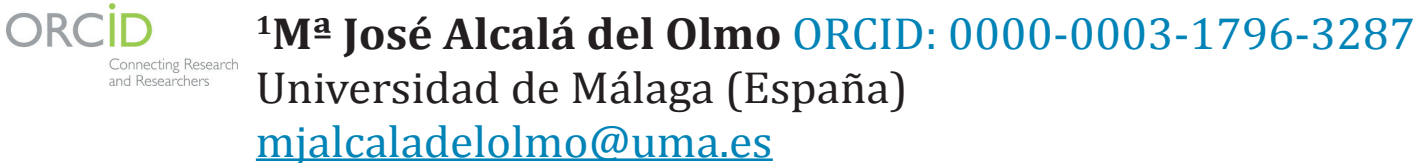

\section{RESUMEN}

Actualmente, asistimos a una serie de profundas transformaciones sociales cuyos efectos se han dejado sentir de una forma patente en los centros de formación superior.

En concreto, el denominado Espacio Europeo de Educación Superior representa un importante desafío para el profesorado universitario, en la medida en que buena parte de sus funciones se han visto notablemente alteradas, al tener que abandonar las clases magistrales de corte tradicional para fomentar la activa participación del alumnado en su proceso de construcción de conocimientos y adquisición de aprendizajes.

Bajo este paradigma, las competencias se convierten en el eje central de la actividad pedagógica, representando el compromiso de ayudar a los estudiantes a desempeñar su profesión con la mayor eficiencia y eficacia posible.

Nos encontramos, así pues, con una universidad que debe abrirse a la vida y sus principales conflictos, conjugando la formación de carácter teórico con una formación práctica que permita asumir compromisos en la resolución de problemáticas sociales, trabajando desde la ética y la responsabilidad.

En este nuevo escenario, el profesor universitario debe poner en marcha y desarrollar nuevas competencias profesionales, lo que, al mismo tiempo, incide de forma significativa en la conformación de su identidad profesional.

A lo largo de este trabajo procederemos a analizar tales competencias, de la misma forma que reflexionaremos en torno a la importancia de la formación, como modo de garantizar que el profesorado llegue a ser capaz de preparar a sus estudiantes del mejor modo posible.

Palabras clave: educación superior; convergencia europea; identidad profesional; formación del docente universitario 


\title{
EST UDIOS Y ENSAYOS
}

\begin{abstract}
At present, we are witnessing a series of deep social transformations whose effects have been felt in a way quite patent in centres of higher education.

In particular, the so-called European area of higher education represents an important challenge for university teachers, as many of its functions have been significantly altered, having to abandon the masterclasses of traditional court to encourage the active participation of students in the process of construction of knowledge and acquisition of learning.

Under this paradigm, the competencies become the central axis of the educational activity, representing the commitment of helping students to carry out their profession with the more efficient and effective as possible.

We are, as well, with a university that should open up to life and its main conflicts, combining the theoretical training with practical training commitments in the resolution of social problems, working from the ethics and responsibility.

In this new scenario, the professor must start and develop new skills, which, at the same time, affects in a significant way in the construction of their professional identity.

Throughout this work, therefore, proceed to analyze such powers, in the same way that we will reflect on the importance of the training designed to provide a superior education of the highest possible quality.
\end{abstract}

Keywords: higher education; european convergence; professional identity; the university teacher training

\section{INTRODUCCIÓN}

El Espacio Europeo de Educación Superior reclama un cambio en la cultura o tradicional modelo de profesor universitario.

Se trata de una apuesta por fomentar un aprendizaje de corte constructivista, en el que el estudiante adquiere pleno protagonismo en su proceso de construcción de conocimientos, encargándose el profesorado de promover su autonomía, dotar de sentido y coherencia a la actuación pedagógica y llevar a cabo un seguimiento planificado de los conocimientos que el alumnado va logrando asimilar y adquirir de forma progresiva.

Dicho Espacio tiene sus antecedentes más significativos en la Declaración de la Sorbona de 1998, la Declaración de Bolonia de 1999, la Conferencia de Salamanca de 2001, la reunión del Consejo de Europa en la Cumbre de Barcelona de 2002 y la Cumbre de Ministros de Berlín de 2003, dirigiendo sus esfuerzos a la homologación de los procesos de formación de las distintas universidades, para favorecer la libre circulación de todos y cada uno de los estudiantes por los entornos laborales de los diversos países de Europa (Mérida, 2013).

El eje central de la docencia universitaria, en este contexto, reside en el alumnado, lo que implica transitar de un modelo de enseñanza de carácter plenamente expositivo a un modelo en el que estudiante, profesorado y contexto interactúan de forma continua.

Resulta determinante, en coherencia con lo anterior, valorar la competencia pedagógica de los docentes universitarios junto a su competencia científica, para llegar a ser capaces de estimular el aprendizaje de todos y cada uno de sus estudiantes. 


\section{ESTUDIOS Y ENSAYOS}

La preparación del alumnado, bajo este paradigma, está fuertemente orientada a favorecer una positiva inserción en el mercado de trabajo.

En este sentido, la llamada Estrategia Europea 2020 reclama la necesidad de que las instituciones de Educación Superior asuman el compromiso y la responsabilidad de formar a los estudiantes en aquellas competencias que hagan posible una efectiva inserción laboral y que favorezcan la empleabilidad, lo que conlleva redefinir en cierta medida la forma de plantear la docencia universitaria (Martínez \& González, 2018).

Para responder a las exigencias y directrices formuladas en ese Espacio Europeo que ya hemos nombrado, también conocido como proceso de Convergencia Europea, es necesario proporcionar una formación destinada al aprendizaje de competencias, siendo éstas el pilar central de la preparación académica ofrecida al alumnado universitario (González \& Wagenar, 2003).

Todo ello conlleva un cambio en la cultura profesional y docente del profesorado, quien ha de abandonar las clases magistrales de corte tradicional para posibilitar que el alumnado construya sus aprendizajes de forma activa y significativa, consistiendo su función más que en transmitir contenidos, en mediar entre la información, los recursos y los diferentes materiales facilitados a sus estudiantes para aprender de forma progresiva (Aznar \& Ull, 2009).

Cuando hablamos de la necesidad de fomentar el desarrollo de competencias en la universidad, estamos haciendo alusión a la relevancia que adquiere el aprendizaje permanente, dado que debemos ofrecer una educación en la que confluyen muy diversas variables: Capacidades, habilidades y actitudes, que se consideran más útiles y prácticas que la mera acumulación de conocimientos (Martínez, Martínez \& Muñoz, 2008).

Nos encontramos, en este nuevo escenario, con un docente que debe responder a un desafío de magnitudes indudables: ofrecer a su alumnado una formación académica y a la vez plenamente unida a escenarios prácticos (Vargas, Pérez de Guzmán \& Bas, 2013), con el objetivo de desarrollar con la mayor eficacia y eficiencia posible un determinado desempeño profesional.

Ambos espacios y realidades, esto es, el educativo y el laboral, edifican sus fundamentos alrededor del concepto de competencia, buscando trascender la noción tradicional de que el conocimiento es el único requisito necesario para poder desarrollar una profesión.

Ahora bien, para asumir este desafío, hacen falta motivaciones, inquietudes, expectativas y valores que lleven al alumnado universitario a tratar de dilucidar cuál es su rol en el contexto socio-laboral en el que desea construir su proyecto de vida, tanto personal como profesional.

Distintos autores reconocen que las universidades actuales tienen una verdadera responsabilidad social (Tejada, 2012; Martínez, 2008), destacando en este sentido la llamada Declaración de Talloires, firmada en Francia en 2005, en la que se delimitaban de forma explícita las funciones cívicas y las responsabilidades sociales que debe asumir la Educación Superior (Tejada, 2013). 


\section{EST UDIOS Y ENSAYOS}

Son dos, fundamentalmente, las razones que llevan a integrar las competencias en el contexto universitario (Yániz \& Villardón, en Tejada \& Ruiz, 2016):

- Se trata de posibilitar el acercamiento de la universidad a su entorno y al marco laboral, siendo esta una Recomendación que se recoge en el Informe Universidad 2000 de la Conferencia de Rectores de las Universidades Españolas para profesionalizar la formación universitaria.

- Constituye un modo de reclamar una enseñanza más práctica y vinculada a entornos reales, capaz de favorecer en los estudiantes la construcción de aprendizajes funcionales, necesarios para desempeñar una profesión de la mejor manera posible.

Atendiendo a los planteamientos anteriores, presentamos este trabajo, cuyo objetivo general se centra en analizar de qué forma incide el llamado proceso de Convergencia Europea en la construcción de la identidad profesional del docente universitario.

Para ello, nos hemos apoyado en una metodología eminentemente descriptiva, puesto que hemos llevado a cabo un proceso de revisión bibliográfica con el que profundizar en torno a cuestiones que definen este trabajo, esto es, las principales competencias profesionales de los docentes universitarios en la actualidad, los desafíos que en su desempeño profesional genera el Espacio Europeo de Educación Superior y la necesaria formación del profesor de universidad, reconociendo en todo momento los efectos positivos que de ella pueden desprenderse para incrementar la calidad de la docencia impartida.

Con las conclusiones y referencias bibliográficas, cerramos las páginas de este trabajo, con el que esperamos tomar conciencia de la complejidad de la labor del docente universitario actual, junto a los compromisos y responsabilidades que este profesional ha de asumir.

\subsection{Objetivos y metodología}

Los objetivos fundamentales en los que se apoya la realización del presente trabajo se resumen de la forma siguiente:

- Reflexionar en torno al Espacio Europeo de Educación Superior, a fin de conocer las principales repercusiones que de él se derivan tanto en el alumnado como en los docentes universitarios.

- Identificar las principales competencias profesionales que debe poseer un profesor universitario en la actualidad.

- Analizar el concepto de identidad profesional y su conformación en los profesores de enseñanza superior, junto a las variables en torno a las que se sustenta dicha identidad profesional.

- Estudiar la relación existente entre formación y configuración de la identidad profesional en los docentes universitarios.

- Delimitar la influencia que tienen las concepciones de los docentes sobre la enseñanza, el aprendizaje y la investigación en la conformación de la identidad profesional. 


\section{ESTUDIOS Y ENSAYOS}

La metodología en la que vamos a apoyarnos es de tipo descriptivo, en la medida en que vamos a centrarnos en llevar a cabo un proceso de revisión documental que nos permita tanto construir un marco teórico, como conocer en profundidad el tema central objeto de estudio.

El profesorado en el Espacio Europeo de Educación Superior: Principales competencias.

\section{EN LOS ÚLTIMOS AÑOS, NUESTRAS UNIVERSIDADES HAN ATRAVESADO CAMBIOS DE GRANDES DIMENSIONES}

De ser espacios destinados de forma exclusiva a transmitir conocimientos, se han convertido en escenarios comprometidos en el abordaje de temáticas de verdadera trascendencia e interés social, al objeto de promover valores y actitudes que lleven a los educandos a asumir compromisos y responsabilidades en su resolución.

Asistimos, además, a un replanteamiento del escenario universitario, motivado por el llamado Espacio Europeo de Educación Superior, que propone una modificación sustancial desde una enseñanza basada en el conocimiento hacia un aprendizaje por competencias, destinadas a propiciar en el estudiante una efectiva integración en el entramado socio-laboral.

Además, es importante que pongamos de manifiesto que dicho Espacio Europeo se articula en torno a los fundamentos básicos que presentamos a continuación (Cifuentes, 2006):

- Transparencia de los procesos formativos, lo que implica centrar la atención de forma prioritaria en los procesos de aprendizaje, siendo el propio aprendizaje el eje central del proceso formativo, la interacción didáctica y el diseño curricular.

- Dicha transparencia podrá hacerse realidad en la medida en que exista una clara definición de las tareas que se proponen a los estudiantes en su valoración correspondiente en términos de créditos.

- Es fundamental lograr una efectiva transferencia de aprendizajes, lo que supone delimitar de forma específica los objetivos de la asignatura junto a la calificación final.

- Tanto la transparencia como la transferencia de aprendizajes genera importantes repercusiones docentes y obligan a reconceptualizar buena parte de sus funciones y su identidad profesional.

En este nuevo escenario, el docente se convierte en un impulsor y facilitador del aprendizaje de todos y cada uno de sus estudiantes, así como de las competencias y actitudes que éstos deben adquirir, dejando así de ser un mero transmisor de conocimientos.

Son muy diversos los factores que ayudan a comprender la necesidad de insertar las competencias en el marco de la formación superior (Villardón-Gallego, Yániz, Achurra, Iraurgi \& Aguilar, 2013):

- Se pretende favorecer la vinculación entre universidad y realidad laboral, con objeto de profesionalizar la formación ofrecida. 


\section{EST UDIOS Y ENSAYOS}

- Constituye un modo de reclamar una enseñanza más funcional, conectada con espacios reales y sus principales demandas y necesidades.

- Ayuda a llevar a cabo un continuo replanteamiento y redefinición de aquellos aprendizajes que resultan realmente determinantes y que han de asegurarse al alumnado universitario.

Todo ello plantea, a su vez, la necesidad de recurrir a nuevas propuestas metodológicas, que concedan pleno protagonismo al alumnado y le conviertan en eje central de la actividad pedagógica.

En coherencia con lo anterior, resulta necesario recurrir a métodos mucho más dinámicos, favorecedores de una mayor interacción entre estudiantes y entre éstos y los docentes, de la misma forma que resulta fundamental recurrir a las Tecnologías de la Información y la Comunicación.

En este escenario, las competencias del profesorado universitario resultan determinantes, y se pueden definir como el conjunto de aquellos conocimientos, habilidades, valores y actitudes que permiten que el docente llegue a desarrollar una docencia de verdadera calidad, con capacidad para ofrecer respuesta a los problemas que puedan desencadenarse de forma progresiva (Bozu \& Canto, 2009).

Autores de la talla de Zabalza (2011), reconocen que la identidad y el desarrollo profesional del profesorado universitario ha de sustentarse en la progresiva adquisición y dominio de las competencias siguientes:

- Planificación adecuada del proceso de enseñanza y aprendizaje, lo que implica decidir previamente a la entrada en el aula aquello que se va a trabajar y de qué modo, con objeto de conceder un hilo conductor al discurso y llenar de pleno sentido y coherencia la actuación y el quehacer pedagógico.

Planificar significa tener en cuenta una serie de variables como son los descriptores en los que se apoya y sustenta la asignatura que se ha de impartir, los contenidos fundamentales de la misma, el plan de estudios o espacio curricular en el que se enmarca esta, el curso y la duración, las características del alumnado con el que trabajar, la ratio, así como la propia imagen que tiene el profesorado sobre la disciplina y su didáctica (Zabalza, 2006).

De la misma forma, durante el proceso de planificación, el docente universitario debe delimitar aquellas competencias que va a tratar de promover y trabajar con sus estudiantes en el marco de la asignatura que se encarga de impartir. Para ello, debe mantener relaciones con el ámbito laboral, a fin de ofrecer un acercamiento al mundo real y tener en cuenta los retos sociales a los que hay que tratar de ofrecer respuesta con la formación universitaria ofrecida (Mérida, González \& Olivares, 2012).

La planificación, en coherencia con todos los planteamientos que hemos expuesto resulta determinante, lo que nos lleva a su vez a reflexionar en torno a lo que autores como Bain (2007), reconoce como profesores excepcionales durante ese proceso de preparación de sus clases: 


\section{ESTUDIOS Y ENSAYOS}

Los profesores excepcionales tratan sus clases, sus discusiones programadas, sus sesiones de resolución de problemas y demás elementos de su enseñanza como esfuerzos intelectuales formales que son intelectualmente exigentes y tan importantes como su investigación y su trabajo académico. Esa actitud es quizás más patente en las respuestas que nuestros sujetos proporcionan a una cuestión sencilla: “¿Qué te preguntas cuando te dispones a preparar tu docencia?”. En algunos profesores esta solicitud de información podría haber sugerido respuestas poco inspiradas que enfatizasen lo prosaico: ¿Cuántos estudiantes tendré? ¿Qué incluiré en mis clases? ¿Cuántos exámenes les haré y de qué tipo? ¿Qué les daré para que lean? (...) Nuestros sujetos utilizan una serie de preguntas mucho más rica a la hora de diseñar una clase, una conferencia, una discusión, unas prácticas profesionales o cualquier encuentro con estudiantes, y comienzan con cuestiones sobre los objetivos de aprendizaje para los estudiantes, en lugar de con aquéllas que plantean qué debe hacer el profesor (Bain, 2007, p. 28).

A la vista de los planteamientos anteriores, podemos reconocer sin lugar a dudas la relevancia de la planificación para ofrecer una docencia de calidad, junto a la importancia de prestar atención a cuestiones relacionadas con el alumnado para llegar a preparar de la forma más eficiente posible las clases por parte del profesorado universitario.

- Selección y preparación de contenidos, teniendo en cuenta aquellos que tengan mayor trascendencia en el marco disciplinar, procurando situarlos al alcance del alumnado, a fin de que éste llegue a ser capaz de asimilarlos de forma satisfactoria.

- Ofrecer información comprensible, que permita que el estudiante llegue a establecer relaciones entre conocimientos y desarrollar aprendizajes verdaderamente funcionales y significativos.

Manejo didáctico de las TICs, como herramientas imprescindibles para hacer la enseñanza más amena, atractiva y de mayor alcance para el alumnado.

- Tutorización, como instrumento pedagógico de gran trascendencia para ofrecer un verdadero acompañamiento y una enseñanza personalizada, ofrecer respuesta a la diversidad y conocer aspectos destacados tanto de los estudiantes a los que atender, como de las posibles dificultades con las que éstos se encuentran en el proceso de aprendizaje.

Entendemos la tutoría como la supervisión que el docente realiza de su alumnado, prestando atención al trabajo que éste desarrolla para responder plenamente a sus necesidades e intereses. En su faceta como tutor, el profesor universitario ha de ser un profesional cercano y accesible, con capacidad para estimular una verdadera participación y entusiasmo de su alumnado (Monreal, 2000, en Zabalza, 2006).

De la misma forma, es importante que destaquemos que la tutoría constituye un instrumento fundamental para orientar los aprendizajes del alumnado y ayudarle plenamente a la construcción de sus conocimientos, siendo prioritario planificar y organizar de forma adecuada la misma para propiciar la consecución de los objetivos que esta competencia de los profesores universitarios plantea (Mas \& Olmos, 2012). 


\section{EST UDIOS Y ENSAYOS}

- Capacidad para establecer relaciones constructivas con el alumnado, como forma de que éstos desarrollen una actitud positiva hacia el aprendizaje y se impliquen de forma efectiva.

- Reflexión e investigación centrada en la propia enseñanza, a fin de delimitar posibles aspectos que podrían mejorarse, para enriquecer la praxis profesional.

- Implicación institucional, lo que supone llevar a cabo un trabajo colegiado o compartido con el resto del profesorado.

- Evaluación de los aprendizajes adquiridos por los estudiantes, prestando especial atención a las dificultades que éstos puedan presentar, lo que sirve de fundamento necesario para reorientar la práctica profesional.

En relación con la evaluación, debemos destacar que se trata de superar la tradicional evaluación de carácter sumativo, para promover una continua evaluación de los aprendizajes adquiridos de forma progresiva por el alumnado, valorando tanto sus avances como las posibles dificultades, al objeto de reorientar la intervención pedagógica de la forma más adecuada posible.

El llamado Espacio Europeo de Educación Superior supone, además, personalizar la evaluación a las características del alumnado, buscando ofrecer siempre la mayor calidad posible, recabar la máxima información sobre su proceso de aprendizaje y emplear diversos instrumentos con los que llegar a una toma de decisiones coherente en relación con la calificación a otorgar.

De la misma forma, debemos destacar que es fundamental que el docente universitario establezca desde un principio y de forma absolutamente explícita al alumnado de qué forma se le va a evaluar, buscando el consenso y superando todo tipo de temores e incertidumbres que este tema pueda llegar a suscitar.

- Gestionar metodologías de trabajo didáctico y tareas de aprendizaje, buscando satisfacer las necesidades e intereses del alumnado e incrementar su motivación.

A este respecto, Mérida (2013), considera que la lección magistral que durante años ha persistido en la universidad, deja de tener tanta relevancia en este nuevo contexto, encargándose ahora el docente de configurar su aula desde un enfoque investigador, que lleve al estudiante al cuestionamiento, la reflexión y la crítica, e incluso a la búsqueda de respuesta a ciertas hipótesis que puedan plantearse.

Atendiendo a la presencia de alumnado cada vez más diverso en las aulas universitarias, se hace necesario poner en marcha diversas estrategias metodológicas, que consigan responder a la diversidad en un planteamiento de universidad inclusiva e integradora.

Estrategias como debates, grupos de discusión, talleres, seminarios y mesas redondas pensamos que cobran verdadero sentido.

- Trabajar desde la ética profesional (Rodríguez, 2003), lo que implica asumir compromisos de naturaleza tanto social como institucional, cumplir con las respon- 


\section{ESTUDIOS Y ENSAYOS}

sabilidades y compromisos que se delimitan en su contrato de trabajo y ser justo en las valoraciones proporcionadas al alumnado.

Para finalizar con estas cuestiones, nos parece interesante recurrir a las aportaciones de autores como Valcárcel (2003), que define que además de las competencias que hemos señalado anteriormente, en el actual escenario universitario el docente ha de reunir las competencias básicas que recogemos a continuación:

Competencias cognitivas relacionadas con la asignatura concreta que se encarga de impartir, con el fin de llegar a ser capaz de transmitir información relevante relacionada con la misma a sus estudiantes, lo que a su vez le permitirá profundizar en cuestiones específicas y resolver dudas y problemas que puedan plantearse.

- Competencias metacognitivas, con las que llegar a repensar la actuación pedagógica, reflexionando de forma constante sobre la misma, para llegar a perfeccionarla de forma continua.

- Competencias comunicativas, necesarias para favorecer la asimilación y comprensión de los contenidos en los estudiantes. Estas competencias deben ir en paralelo a las competencias cognitivas, en la medida en que el docente puede ser gran conocedor e incluso experto en su materia, pero si carece de las habilidades necesarias para transmitirla, el alumnado tendrá verdaderas dificultades para dotar de sentido y significado a aquello que se está trabajando en el aula.

- Competencias sociales, que le permitan desarrollar un trabajo colegiado y coordinado con el resto del profesorado, para evitar repeticiones y solapamientos en las cuestiones trabajadas en las aulas, necesario además para emprender actuaciones interdisciplinares.

- Competencias emocionales, necesarias para trabajar desde el respeto, la tolerancia y empatía con sus estudiantes, buscando siempre ajustar su intervención a las necesidades e intereses de estos, así como al objeto de ofrecer respuesta a las dificultades con las que se puedan encontrar.

\section{LA IDENTIDAD PROFESIONAL DE LOS DOCENTES UNIVERSITARIOS EN EL MARCO DE LA CONVERGENCIA EUROPEA}

Llegar a convertirse en profesor de universidad y configurar o construir la propia identidad no es algo que se produzca de golpe o de un modo un tanto brusco, sino que es el resultado de un proceso de socialización que tiene lugar en la institución en la que se desempeña el ejercicio profesional.

La forma en la que el docente va transformándose para llegar a ser profesor de universidad es el resultado de un proceso de desarrollo personal y profesional, que integran el ambiente de trabajo en la institución junto con las características concretas de la profesión docente constituida históricamente (Gonçalves, 1996, en Gewerc, 2001).

Desde un planteamiento general, podemos reconocer que la identidad del docente universitario y sus propias concepciones sobre su profesión se articulan en torno a dos de sus funciones principales, esto es, la docencia y la investigación. 


\section{EST UDIOS Y ENSAYOS}

En el caso de la docencia, nos parece interesante destacar una serie de investigaciones emprendidas por diferentes autores, en las que se pone de manifiesto que el docente universitario configura su docencia en torno a seis tipos concretos de creencias (Prosser, Trigwell \& Taylor, 1994, en Caballero \& Bolívar, 2015):

- La enseñanza como actividad caracterizada por la transmisión de contenidos delimitados y recogidos en la guía docente.

- La enseñanza como actividad que se centra en transmitir el conocimiento que forma parte del profesorado.

- La enseñanza como proceso de ayuda destinado a que el alumnado pueda asimilar de forma efectiva y adquirir los contenidos reflejados en la guía docente.

- La enseñanza como proceso de apoyo ofrecido al estudiante para llegar a adquirir los conocimientos que pertenecen al profesorado.

- La enseñanza como ayuda ofrecida al alumnado para construir y desarrollar nuevas concepciones.

- La enseñanza como apoyo ofrecido al alumnado para llegar a modificar, ampliar y enriquecer sus convicciones.

En coherencia con lo anterior, podemos reconocer que estas concepciones sobre la enseñanza están estrechamente relacionadas con las diferentes fases o etapas por las que transcurre el desarrollo profesional del docente universitario. Por ello, en consonancia con la mayor adquisición de experiencia en el marco de la docencia, las concepciones del profesorado sobre la enseñanza y la forma en la que los estudiantes acceden al conocimiento van evolucionando, de la misma forma que también evoluciona su propia identidad profesional.

También es importante que reflejemos que la identidad profesional del docente de universidad se relaciona significativamente con sus propias concepciones sobre el aprendizaje.

Así, podemos encontrarnos docentes que definen el aprendizaje como proceso en el que el estudiante es un receptor pasivo de información y de conocimientos, no fomentándose en este caso la reflexión ni la crítica en los procesos de enseñanza, frente a aquellos docentes para los que el aprendizaje debe conceder un pleno protagonismo al alumnado, quien habrá de reflexionar, argumentar y criticar de forma constante aquello que se somete a análisis y asimilación.

La investigación, por otra parte, constituye otra dimensión fundamental en la que los profesores universitarios se apoyan para conforman su identidad profesional.

En este sentido, debemos reconocer que en la enseñanza superior, el docente no debe limitarse de forma exclusiva a impartir sus clases, sino que, además, debe ser investigador, procurando encontrar el equilibrio entre ambas responsabilidades o funciones, teniendo presente que la labor investigadora constituye una herramienta al servicio de la promoción profesional. 


\section{ESTUDIOS Y ENSAYOS}

Aunque la tendencia general suele ser la separación entre docencia e investigación (Caballero, 2009), desde nuestro punto de vista el planteamiento que cobra verdadero sentido es establecer relaciones significativas entre ambas dimensiones, procurando que en la docencia se reflejen resultados de investigaciones emprendidas.

Todo ello pensamos que enriquecerá la enseñanza que el profesor trata de ofrecer a sus estudiantes, de la misma forma que la dotará de mayor sentido y coherencia.

De la misma forma, puesto que somos conscientes de que la investigación constituye una de las tareas principales que forman parte del cometido del profesor de universidad y tiene un peso relevante en su promoción profesional, pensamos que ello no debe significar que el docente de la espalda a la docencia o llegue a descuidarla de alguna forma, puesto que todo ello irá en detrimento de la formación proporcionada a los estudiantes.

De acuerdo con este planteamiento, presentamos una cita de autores como De Vicente \& otros (1996, en Caballero, 2009), en la que un docente pone de manifiesto lo siguiente:

La impresión más fuerte que tuve al acceder a un puesto de profesor de Universidad fue comprobar que la docencia no es lo prioritario en el trabajo del profesor universitario. Me rompió todos los esquemas que tenía como alumno y entendí por qué los profesores no ofrecían toda la dedicación que demandábamos los alumnos (p. 40).

El concepto de identidad profesional, por otra parte, lleva a delimitar cómo el sujeto se define a sí mismo en el plano laboral, es decir, responde al interrogante "¿Quién soy?; ¿a qué me dedico?; ¿cuáles son mis responsabilidades principales?”

Actualmente, se trata de un concepto empleado para dejar constancia de aquellas variables relacionadas con la profesionalidad (Gewerc, 2001).

Cuando analizamos la identidad profesional del profesor de universidad debemos ser conscientes de que es el resultado de un constante proceso de socialización, que tiene lugar a través del propio desempeño y la asimilación de la cultura profesional.

Siguiendo a Caballero (2009), dicha identidad profesional puede analizarse teniendo en cuenta las dimensiones siguientes: Comunidad disciplinar, biografía personal e ideología.

Veamos detenidamente cada una de estas dimensiones.

En lo referente a la relación existente entre identidad profesional y comunidad disciplinar, debemos destacar que dicha comunidad es determinante en tanto que ofrece el lenguaje con el que las personas interactúan y llegan a ser capaces de configurar significados que pueden comprenderse y aplicarse en el ámbito laboral.

En este sentido, podemos señalar que la conformación de la identidad profesional del profesor de universidad se genera en un contexto caracterizado por la existencia de relaciones de poder, en las que las diferentes comunidades disciplinares van desarrollando identidades con las que extender y racionalizar su dominio. 


\section{ESTUDIOS Y ENSAYOS}

La identidad profesional, además, se relaciona de forma significativa con la biografía personal, definida como el conjunto de experiencias y la trayectoria vital que envuelve al profesor universitario.

Así, sus vivencias, experiencias personales y acontecimientos relevantes a lo largo de su vida inciden de forma poderosa en la conformación de su identidad profesional.

De la misma forma, podemos establecer relaciones entre la conformación de esta identidad y la ideología; esto es, el docente cuenta con una serie de valores, creencias e incluso expectativas, que se vislumbran en su propia práctica y le ayudan a conformar su propia identidad.

Asumir una identidad profesional, además, supone conjugar tres componentes específicos (Caballero, 2009):

- Dimensión externa, impuesta por la profesión misma, en la que se incluyen funciones y características que hacen que podamos diferenciar nuestra profesión de otras.

- Dimensión interna, entendida como el modo de comprender la profesión y ponerla en práctica.

- Dimensión interactiva, teniendo en cuenta las relaciones que se establecen durante el desempeño profesional.

La identidad profesional influye de forma relevante en la vida personal y social de cada uno de nosotros.

Teniendo en cuenta que aquello que hacemos conforma en gran medida nuestra forma de ser, podemos señalar que las personas nos comprendemos mejor a nosotras mismas en función de las tareas profesionales que desarrollamos.

La profesión, así concebida, nos ofrece una perspectiva personal y un modo concreto de entender y de enfocar nuestra vida.

La identidad personal constituida por aquello que somos, aquello que pensamos y aquello que hacemos está, así pues, innegablemente relacionada con la labor profesional que desarrollamos.

En el caso de los profesores universitarios, podemos reconocer que éstos van conformando su identidad profesional durante el transcurso de su formación inicial, encargándose en ese proceso de construir todo un conjunto de saberes y un saber hacer o proceder, así como en la interiorización de dichos saberes en un "saber ser" (Giddens, 1995, en Bolívar, Fernández-Cruz \& Molina, 2005), que llevan a identificar a este profesional como una persona que se construye a partir de múltiples experiencias vitales.

El concepto de identidad, en coherencia con los planteamientos que hasta ahora hemos expuesto, presenta un carácter plenamente dinámico y cambiante, que pone de manifiesto la existencia de una cierta estabilidad en el tiempo, y es producto de la interacción con los demás en un proceso de continua construcción y reconstrucción. 


\section{EST UDIOS Y ENSAYOS}

A lo largo de este proceso, el profesor llega a desarrollar todo un conjunto de creencias de naturaleza pedagógica que guían su forma de intervenir en el aula y responden a un contexto social y educativo en el que se encuentra inmerso (González, Río \& Rosales, 2002).

Podemos reconocer que el docente llega a disponer de una identidad profesional consolidada una vez que toma conciencia de cuáles son sus creencias implícitas sobre sí mismo y los demás. Ello nos permite afirmar que las concepciones que el profesor tiene de los demás junto con su propia forma de percibirse a sí mismo desempeñan un papel fundamental para adquirir una identidad tanto personal como profesional.

Existen diferentes variables que inciden de forma significativa tanto en la conformación de la identidad profesional en los docentes que ejercen sus funciones en la Educación Superior, como en su propio desarrollo profesional.

Veamos detenidamente cada uno de ellas:

\begin{tabular}{|l|l|}
\hline VARIABLES & INFLUENCIA EN LA IDENTIDAD Y EN EL DESARROLLO PROFESIONAL \\
\hline Profesionales & $\begin{array}{l}\text { Se vinculan con la ejecución y desempeño de la profesión. Entre estas variables } \\
\text { podemos destacar los apoyos recibidos de forma institucional, recursos materiales } \\
\text { y humanos con los que trabajar de forma efectiva, ambiente laboral, salario, } \\
\text { reconocimientos, etc. }\end{array}$ \\
\hline Personales & $\begin{array}{l}\text { Se refieren a todos aquellos que forman parte del profesor y le constituyen como } \\
\text { persona: rasgos de personalidad, estado físico y emocional, variables cognitivas, } \\
\text { edad, factores motivacionales, formación, etc. }\end{array}$ \\
\hline Contextuales & $\begin{array}{l}\text { Se trata de elementos que influyen en el profesor, pese a ser externos a él, tales } \\
\text { como el contexto familiar, relaciones establecidas, etc. }\end{array}$ \\
\hline Sociales & $\begin{array}{l}\text { Constituyen factores externos incidentes significativamente en la vida profesional } \\
\text { del docente: acontecimientos de índole social, política, económica y cultural. }\end{array}$ \\
\hline
\end{tabular}

Tabla 1. Variables que influyen en la identidad y desarrollo profesional

Fuente: Elaboración personal a partir de Caballero \& Bolívar (2015)

En definitiva, a la vista de los planteamientos que hemos expuesto para reflexionar en torno a la identidad del docente universitario, podemos reconocer que en el proceso de construcción de dicha identidad inciden muy diversas variables, entre las que ocupa un peso relevante tanto la docencia como la investigación.

El cierto desequilibrio o desajuste entre ambas dimensiones e incluso el predominio de la investigación sobre la docencia, influirá en una lenta adquisición de aprendizajes por parte del alumnado.

Podemos concluir afirmando, tal y como sostiene Zabalza (2009), que el profesor de universidad tiene una identidad doble, en tanto que, de la misma forma que es especialista en su campo científico, también lo es en el desarrollo de procesos de enseñanza y aprendizaje de la disciplina que se encarga de impartir. 


\section{LA NECESARIA FORMACIÓN DEL DOCENTE UNIVERSITARIO: SUS RELACIONES CON LA IDENTIDAD PROFESIONAL}

Como en la mayor parte de los países, en España la formación del profesorado universitario es de carácter voluntario y gratuito, no siendo necesario para poder enseñar recibir una formación pedagógica previa (Carballo, 2016).

Antes de comenzar a analizar la importancia de la formación en los profesores de universidad, pensamos que es imprescindible reflexionar en torno a algunas de las características definitorias de los docentes que ejercen sus funciones en este contexto, al ser diferentes de aquellos que desempeñan su labor en el resto de niveles educativos.

1) En primer lugar, debemos destacar que a lo largo de su proceso de formación, los profesores de universidad conceden gran prioridad a su propio ámbito disciplinar.

2) Por otra parte, este profesional está más orientado a la disciplina que a su profesión docente, conjugando tareas no sólo centradas en la docencia, sino también en la investigación.

3) Además, debe atender a estudiantes caracterizados por una marcada heterogeneidad, lo que se refleja tanto en la edad, como en las motivaciones, intereses, expectativas, experiencias y capacidades.

4) Finalmente, podemos señalar que debe trabajar en un contexto en el que se concede gran protagonismo al estudiante en la gestión de su proceso formativo, subrayándose la necesaria autonomía que ha de adquirir el mismo, unida a la capacidad de autorregulación de los aprendizajes.

Las características del profesorado universitario, así pues, son determinantes, en tanto que constituyen un marco de referencia decisivo para diseñar la formación que hay que proporcionarles.

Se trata, tal y como apuntan Zabala, Cid \& Trillo (2014), de personas adultas y con cierta madurez, es decir, con altas capacidades intelectuales, que incluso se perciben e identifican lo suficientemente formadas en sus ámbitos disciplinares.

Siguiendo a los autores que acabamos de apuntar, la oferta formativa que las universidades tratan de proporcionar al profesorado universitario presenta, además, las características que recogemos a continuación:

1) Por una parte, debemos destacar que en la mayor parte de los casos, se plantea como una actividad voluntaria, e incluso no relacionada con el marco disciplinar al que se pertenece.

2) Por otra, se trata de una oferta que se dirige más a ofrecer respuesta a las necesidades manifestadas por el profesorado, que a la satisfacción de demandas objetivas de la propia institución o incluso relacionadas con el proceso de formación del alumnado, lo que supone que esté en cierta medida desvinculada de los procesos orientados a incrementar la calidad de la docencia. 


\section{ESTUDIOS Y ENSAYOS}

3) Además, dicha oferta formativa tiene escaso peso en el currículum, siendo por ello un instrumento de poca relevancia en relación con la promoción profesional, lo que hace que sea muy bajo el número de docentes que realmente se implican y participan en las iniciativas de formación que tratan de ponerse en marcha en las universidades.

Una vez señalado lo anterior, cabe plantearnos la cuestión siguiente: “¿Cómo contribuye la formación a la construcción de la identidad profesional por parte de los profesores universitarios?"

En principio, debemos señalar que los procesos de formación docente sitúan el eje de la configuración de la identidad del profesorado en su trabajo como docentes, lo que implica reorientar o trasladar sus preocupaciones e inquietudes desde el marco de la especialidad científica en la que fueron formados hacia el ámbito del aprendizaje de los estudiantes.

De la misma forma, suele ser en el proceso de formación inicial en el que el profesorado construye su identidad; esto es, el sentido que cada profesional concede a su formación se relaciona de forma significativa con las motivaciones e intereses que le llevan a aprender e interactuar con otros profesionales. Todo ello hace que el profesor ponga en marcha todo un conjunto de estrategias adecuadas y relacionadas con su trayectoria profesional, así como con las motivaciones que lo llevan a formarse, siendo precisamente éste el eje central de la configuración de su proceso de identidad profesional (Martín- Gutiérrez, Conde-Jiménez \& Mayor- Ruiz, 2014).

Además, podemos señalar que la conformación de la identidad profesional constituye un proceso de construcción individual resultante de la interacción que se establece con otros profesionales, por lo que presenta una vertiente socializadora, debiendo la universidad fomentar la creación de espacios y encuentros para que esa construcción sea posible (Zabalza, 2011).

En general, el profesorado que ya ha tenido alguna experiencia formativa, siente la necesidad e interés en continuar ampliando y enriqueciendo su formación (Zabala, Cid \& Trillo, 2014).

Por el contrario, aquellos profesores que no la han tenido, prácticamente no sienten la necesidad ni el impulso de participar en cursos de formación ni la demandan.

Por otra parte, diversas investigaciones han demostrado que aquellas universidades implicadas en la puesta en práctica de experiencias formativas destinadas al profesorado novel, son las que terminan consiguiendo consolidar planes de formación estables. En concreto, dichos estudios ponen de manifiesto la importancia de llevar a cabo un proceso de acompañamiento de los profesores noveles, tanto en el proceso de construcción de su identidad, como en el mismo proceso en el que reciben formación (Gewerc, 2011, en Martín- Gutiérrez, Conde-Jiménez \& Mayor- Ruiz, 2014).

La implicación de las universidades en la promoción de dicha formación es, pues, determinante para que llegue a incrementarse tanto el interés como la demanda de la propia formación (Austin \& Mc Daniels, 2007, en Zabala, Cid \& Trillo, 2014). 


\section{EST UDIOS Y ENSAYOS}

Constituye, así pues, un elemento central al servicio de la calidad de la enseñanza. A este respecto, De Miguel (2003), subraya la necesidad de regular políticas de carácter institucional destinadas a promover el desarrollo profesional docente, cuyo eje de actuación resumimos de la forma siguiente:

- Por un lado, resulta necesario delimitar con claridad los distintos criterios que nos ayudan a identificar hasta qué punto la función docente cuenta con criterios de excelencia.

- Por otro, es importante contar con programas debidamente acreditados y destinados a la formación pedagógica de los docentes universitarios.

- A todo lo anterior podemos sumar la relevancia de establecer pruebas con la suficiente transparencia, cuya superación permitirá contratar y seleccionar al profesorado.

El diseño de programas formativos destinados al profesorado universitario debe reunir las características que recogemos a continuación (Martín- Gutiérrez, CondeJiménez \& Mayor- Ruiz, 2014):

- Es fundamental ofrecer respuesta a las necesidades detectadas y sentidas por el propio colectivo profesional.

- Resulta determinante propiciar la formación en el mismo lugar de trabajo, de forma que incluso pueda recibirse aprendizajes proporcionados por otros compañeros de profesión.

- Es necesario que la oferta formativa se caracterice por una total flexibilidad, combinando incluso la formación de carácter presencial o semipresencial, a fin de ofrecer a todos los docentes la posibilidad de participar en la misma, sin que ello entorpezca su rutina y sus compromisos diarios con el ejercicio profesional.

Se trata, en definitiva, de ayudar a superar temores e incertidumbres, de configurar espacios de trabajo compartido y de tener incluso acceso al conocimiento de experiencias emprendidas en la universidad en el marco de la innovación docente, a fin de llegar a aplicarlas en diferentes contextos y situaciones.

La formación, así pues, debe ser un instrumento que ayude al profesorado a reflexionar y analizar su propia práctica, a compartir experiencias con otros compañeros y a tratar de construir una identidad profesional coherente y firme, que permita valorar con cierta claridad a qué nos dedicamos, cómo podemos mejorar y cómo enriquecer nuestra práctica profesional gracias a las aportaciones de otros docentes, con más experiencia e incluso con cierta formación didáctica.

\section{CONCLUSIONES}

A la vista de los planteamientos sobre los que hemos reflexionado a lo largo de las páginas que conforman este trabajo, reconocemos que nuestras actuales universidades han cambiado de forma significativa. 


\section{ESTUDIOS Y ENSAYOS}

Ello obliga a un replanteamiento de las funciones docentes, así como a una reformulación de la propia formación pedagógica que ha de proporcionarse al profesorado universitario.

Nos encontramos, además, con un nuevo contexto socio-laboral, en el que la presencia de nuevas necesidades sociales da lugar al desarrollo de nuevas profesiones, lo que hace necesario dotar al estudiante universitario de herramientas suficientes con las que convertirse en un profesional eficaz y eficiente.

El profesorado universitario debe trabajar, así pues, para facilitar en el alumnado el reconocimiento de nuevas oportunidades laborales, la aceptación de desafíos, la toma de conciencia de las capacidades propias, la resolución eficaz de los problemas y el desarrollo de un pensamiento sistémico.

Nuestra universidad, así pues, debe "abrirse a la vida y formar para la vida”. Ello supone convertir las aulas de centros universitarios en espacios de indagación, crítica, diálogo, debate, argumentación y cuestionamiento, todo lo cual contribuirá al desarrollo integral del alumnado y la conformación de su personalidad.

El estudiante, así pues, ocupa un lugar activo; es el protagonista principal en el acceso al conocimiento y el docente debe llevar a cabo una función caracterizada por el acompañamiento, ayudando en la construcción de proyectos de vida, siendo este un desafío de grandes dimensiones.

Las aulas universitarias, en este orden de cosas, no pueden ser lugares en los que predominen los monólogos, sino, por el contrario, deben ser espacios en los que se incite al alumnado a un diálogo constructivo, enseñándoles a pensar, desarrollando sus habilidades de cuestionamiento, siento todos ellos aspectos fundamentales para incrementar la motivación e interés hacia los aspectos trabajados, así como fuente principal de aprendizaje que no podemos ni debemos cuestionar.

De la misma forma, queremos destacar la importancia y la necesidad de trabajar desde la más absoluta humildad, respetando los puntos de vista y opiniones del alumnado, a fin de propiciar la profundización en determinados conceptos y trabajar las habilidades sociales con todos ellos.

Son necesarias, así pues, nuevas y desafiantes competencias profesionales, que no sólo implican transmitir contenidos, evaluar aprendizajes y seleccionar diferentes estrategias metodológicas, sino que se trata de llegar aún más lejos, planificando las clases de forma adecuada, llevando a cabo una acción tutorial caracterizada por la cercanía y la accesibilidad y detenerse a reflexionar y analizar la propia práctica.

En relación con la planificación, queremos destacar que la entendemos y definimos no como algo rígido, sino plenamente flexible y dinámico, que ha de ir adaptándose a las circunstancias que se vayan desencadenando, en coherencia con las intervenciones del alumnado, sus dudas, el cuestionamiento en relación con determinados conceptos, etc.

En ese contexto de trabajo que acabamos de describir, el docente universitario debe construir su identidad profesional, conjugando la docencia y la investigación, y pro- 


\section{EST UDIOS Y ENSAYOS}

curando que haya un verdadero equilibrio entre ambas dimensiones, en pro de una enseñanza de la mayor calidad posible.

Debemos tener en cuenta, al mismo tiempo, que la identidad profesional es algo que se construye de forma procesual y en interacción con los demás, presentando por ello una evidente dimensión socializadora.

En esa identidad, además, incide de forma significativa tanto la historia personal de cada individuo y sus experiencias vitales, como los valores, las creencias, las expectativas y el contexto en el que se desarrolla la actividad profesional.

En la mayor parte de los casos, la identidad profesional va conformándose a lo largo de la formación inicial; proceso en el que el docente va tomando conciencia de cuáles son sus principales compromisos y responsabilidades y qué se espera de él.

Pese a no tener carácter obligatorio, consideramos que la formación del docente universitario reviste gran valor, en tanto que puede ayudarle a superar buena parte de dificultades, compartir experiencias y enriquecerse con las aportaciones de otros compañeros de profesión.

Somos plenamente conscientes de que la carrera docente en la universidad constituye un largo proceso, en el que hay que adquirir gran experiencia tanto docente como investigadora, lo que a su vez se relaciona con la posibilidad de obtener finalmente cierta estabilidad profesional.

Destacar, en coherencia con lo anterior, que puesto que se trata de un largo y complicado proceso, es fundamental el componente vocacional; si no amamos de verdad aquello a lo que nos dedicamos y sacrificamos nuestro tiempo en mejorar día a día, el proceso sería aún más complejo y carente de sentido.

\section{REFERENCIAS}

Aznar, P. \& Ull, M.A. (2009). La formación de competencias básicas para el desarrollo sostenible: El papel de la Universidad. Revista de Educación, número extraordinario, 219-237.

Bain, K. (2007). Lo que hacen los mejores profesores de universidad. Valencia. Universidad de Valencia.

Bolívar, A., Fernández -Cruz, M. \& Molina, E. (2005). Investigar la identidad profesional del profesorado: una triangulación secuencial. Forum: Qualitative Social Research, 6 (1), recuperado de https://www.qualitative-research.net/fqs

Bozu, Z. \& Canto, P.J. (2009). El profesorado universitario en la sociedad del conocimiento: competencias profesionales docentes. Revista de Formación e Innovación Educativa Universitaria, 2, (2), 87-97.

Caballero, K. (2009). Construcción y desarrollo de la identidad profesional del profesorado universitario. Tesis Doctoral. Universidad de Granada.

Caballero, K. \& Bolívar, A. (2015). El profesorado universitario como docente: hacia una identidad profesional que integre docencia e investigación. Revista de Docencia Universitaria, 13, (1), 57-77. 
Carballo, R. (2016). La formación del profesorado universitario: una perspectiva a través de la oferta formativa y los responsables de la formación. Deutschland. EAE.

Cifuentes, P. (2006). El profesor universitario ante el Espacio Europeo de Educación Superior. Revista Galego-Portuguesa de Psicoloxía e Educación, 11-12, (13), 43-57.

De Miguel, M. (2003). Calidad de la enseñanza universitaria y desarrollo profesional del profesorado. Revista de Educación, 331, 13-34.

Gewerc, A. (2001). Identidad profesional y trayectoria en la universidad. Profesorado. Revista de Currículum y Formación de Profesorado, 5, (2), 1-15.

González, S., Río, E. \& Rosales, S. (2002). El currículum oculto en la escuela. Buenos Aires. Lumen Humanitas.

González, J. \& Wagenar, R. (2003). Tunning Educational Structures in Europe. Informe Final. Bilbao. Universidad de Deusto.

Martín-Gutiérrez, A., Conde-Jiménez, J. \& Mayor-Ruiz, C. (2014). La identidad profesional docente del profesorado novel universitario. Revista de docencia universitaria, 12 (4), 141-160.

Martínez, P. \& González, N. (2018). Las competencias transversales en la universidad: Propiedades psicométricas de un cuestionario. Educación XXI, 21 (1), 231-262.

Martínez, P., Martínez, M. y Muñoz, J.M. (2008). Aprendizaje de competencias en Educación Superior. Revista Galego-Portuguesa de Psicoloxía e Educación, 16(1,2), 195-215.

Martínez, M. (2008). Aprendizaje Servicio y construcción de la ciudadanía activa en la Universidad: La dimensión social y cívica de los aprendizajes académicos, en Martínez, M. (Ed). Aprendizaje Servicio y responsabilidad social de las universidades (pp. 11-26). Barcelona. Octaedro.

Mas, 0. \& Olmos, P. (2012). La atención a la diversidad en la educación superior: Una perspectiva desde las competencias docentes. Revista de Educación Inclusiva, 5, (1), 159-174.

Mérida, R. (2013). La controvertida aplicación de las competencias en la formación docente universitaria. Revista de Docencia Universitaria, 11 (1), 185-212.

Mérida, R., González, M.E. \& Olivares, M.A. (2012). RIECU: Una experiencia de innovación en el Prácticum I del Grado de Infantil de la Universidad de Córdoba. Profesorado. Revista de currículum y formación del profesorado, 3, 447-465, recuperado de http:// www.ugr.es/local/recfpro/rev163COL12.pdf

Rodríguez, S. (2003). Nuevos retos y enfoques en la formación del profesorado universitario. Revista de Educación, 331, 67-99.

Tejada, J. \& Ruiz, C. (2016). Evaluación de competencias profesionales en Educación Superior: Retos e implicaciones. Educación XXI, 19 (1), 17-38- DOI: 10.5944/ educXX1.12175

Tejada, J. (2012). La alternancia de contextos para la adquisición de competencias profesionales en escenarios complementarios de educación superior. Marco y estrategia. Educación XXI, 15(2), 17-40.

Tejada, J. (2013). La formación de las competencias profesionales a través del aprendizaje servicio. Cultura y Educación, 25, (3), 285-294. 


\section{EST UDIOS Y ENSAYOS}

Valcárcel, M. (Coord) (2003). Programa de estudios y análisis destinado a la mejora de la calidad de la enseñanza superior y de la actividad del profesorado universitario. La preparación del profesorado universitario español para la Convergencia Europea en Educación Superior. Córdoba.

Vargas, M., Pérez de Guzmán, M.V., \& Bas, E. (2013). El espacio latinoamericano de educación superior como parte de la mundialización de los estudios superiores. Diálogos Pedagógicos, 22, 11-26.

Villardón-Gallego, L., Yániz, C., Achurra, C., Iraurgi, I. \& Aguilar, M.C. (2013). Learning competence in university: development and structural validation of a scale to measure. Revista de Psicodidáctica, 18(2), 357-374.

Zabalza, M.A. (2006). Competencias docentes del profesorado universitario. Calidad y desarrollo profesional. Madrid. Narcea.

Zabalza, M.A. (2009). Ser profesor universitario hoy. La cuestión universitaria, 5, 68-80.

Zabalza, M.A., Cid, A., \& Trillo, F. (2014). Formación docente del profesorado universitario. El difícil tránsito a los enfoques institucionales. Revista española de Pedagogía, 257, 39-54.

Zabalza, M.A. (2011). Formación del profesorado universitario: mejorar a los docentes para mejorar la docencia. Educaçao, Santa María, 36, (3), 397-424. 